\title{
Primary Genetic Linkage Maps of the Ascidian, Ciona intestinalis
}

\author{
Shungo Kano ${ }^{1,2 *}$, Nori Satoh ${ }^{2}$ and Paolo Sordino ${ }^{1}$ \\ 'Laboratory of Biochemistry and Molecular Biology, Stazione Zoologica 'A. Dohrn', \\ Naples 80121, Italy \\ ${ }^{2}$ Department of Zoology, Graduate School of Science, Kyoto University, \\ Kyoto 606-8502, Japan
}

\begin{abstract}
For whole-genome analysis in a basal chordate (protochordate), we used F1 pseudo-testcross mapping strategy and amplified fragment length polymorphism (AFLP) markers to construct primary linkage maps of the ascidian tunicate Ciona intestinalis. Two genetic maps consisted of 14 linkage groups, in agreement with the haploid chromosome number, and contained 276 and 125 AFLP loci derived from crosses between British and Neapolitan individuals. The two maps covered 4218.9 and $2086.9 \mathrm{cM}$, respectively, with an average marker interval of 16.1 and $18.9 \mathrm{cM}$. We observed a high recombinant ratio, ranging from 25 to $49 \mathrm{~kb} / \mathrm{cM}$, which can explain the high degree of polymorphism in this species. Some AFLP markers were converted to sequence tagged sites (STSs) by sequence determination, in order to create anchor markers for the fragmental physical map. Our recombination tools provide basic knowledge of genetic status and whole genome organization, and genetic markers to assist positional cloning in $C$. intestinalis.
\end{abstract}

Key words: genetic mapping, polymorphism, recombination rate, ascidian, AFLP

\section{INTRODUCTION}

The ascidian Ciona intestinalis is a living descendant of stem chordates, and an excellent model organism to investigate evolution of gene functions and genome structure, with rich genomic and cDNA/EST resources together with an effective system for developmental biology (reviewed by Satoh et al., 2003). For functional analysis, the mutant approach has recently arisen as a powerful means for molecular embryology in Ciona species (Sordino et al., 2001; Jiang et al., 2005). While techniques for insertional mutagenesis and enhancer traps are available in ascidians using the Minos transposon (Sasakura et al., 2003; Awazu et al., 2004) and I-Scel endonuclease (Deschet et al., 2003), spontaneous and chemically induced mutants can provide us with more phenotypes.

Linkage maps are central to positional cloning in $C$. intestinalis, as isolation of genes involved in mutant phenotypes depends on development of genetic infrastructures. However, a high degree of polymorphism and the lack of laboratory strains have caused unstable results during mapping efforts, especially due to primer incompatibility of genetic markers established in non-coding regions such as microsatellites. To solve these issues, the amplified fragment length polymorphism (AFLP; Vos et al., 1995) technique was chosen. The AFLP technique has been widely used to construct primary genetic maps in many organisms, such as medaka (Naruse et al., 2000), Pacific oyster (Li and

\footnotetext{
${ }^{*}$ Corresponding author. Phone: +39-081-5833283; Fax : +39-081-7641355; E-mail: kano@szn.it
}

Guo, 2004), and plants (Eucalyptus: Myburg et al., 2003; Arabidopsis: Peters et al., 2004). Since C. intestinalis contains high levels of polymorphisms among local populations (Procaccini et al., 2000; Kano et al., 2001; Boffelli et al., 2004), we applied the F1 pseudo-testcross strategy that takes advantage of parental heterozygosity (Grattapaglia and Sederoff, 1994), instead of generating F2 intercrosses or backcrosses. This strategy offered a solution for PCR failure caused by polymorphisms. Recently, pseudo-testcross combined with AFLPs has been applied to aquatic animals, the Pacific oyster (Li and Guo, 2004) and the channel catfish (Liu et al., 2003), allowing quick mapping. Conversion from AFLPs to sequence tagged sites (STSs) may provide us with co-dominant markers (Meksem et al., 2001), which enables researchers to share anonymous markers like AFLPs. This approach can establish entry gates for in silico explorations of candidate genes in the Ciona genome database and anchor loci for assembly of the genome, which still consists of fragmental scaffolds due to polymorphisms (Dehal et al., 2002). Using genetic variability, we report the first construction of two genome-wide linkage maps of the model species $C$. intestinalis as a new platform to advance Ciona genetics and genomics.

\section{MATERIALS AND METHODS}

Crossing panel for linkage maps

Individuals derived from British (southern Plymouth, United Kingdom) and Neapolitan (Fusaro lagoon, Naples, Italy) populations were used for crossing panels by virtue of their genetic differences (see Results). F1 progeny were obtained by crossing eggs from a wild British individual with sperm from a wild Neapolitan individual, since this combination showed no significant difference in fertilization ratio if compared with a reciprocal cross. Fifty speci- 
mens were used for the pseudo-testcross F1 mapping. Animals were reared according to Cirino et al. (2002) and Sasakura et al. (2003), with some modifications.

\section{DNA isolation}

Genomic DNA was extracted as previously described (Kano et al., 2001), with minor modifications. Each tissue from the body wall muscle of an adult specimen was lysed for $4 \mathrm{hr}$ in $0.4 \mathrm{M} \mathrm{NaCl}, 10$ $\mathrm{mM}$ Tris- $\mathrm{HCl} \mathrm{pH}$ 8.0, $2 \mathrm{mM}$ EDTA, 2\% SDS, and $8 \mathrm{M}$ Urea, and incubated at $95^{\circ} \mathrm{C}$ for $10 \mathrm{~min}$. After ethanol precipitation, the CTAB procedure was carried out at $37^{\circ} \mathrm{C}$ instead of $65^{\circ} \mathrm{C}$ to avoid loss of yield, as suggested by Shahjahan et al. (1995). The extracted DNA was of high molecular weight and good for restriction enzymes digestions in the AFLP procedure.

\section{AFLP fingerprints}

AFLP fingerprints were generated using the AFLP Analysis System II kit (Invitrogen), Taq DNA polymerase (5 units/ $\mu$, Roche Applied Science), and a PCR Express DNA thermal cycler (Hybaid) according to manufacturers' instructions. For selective amplifications, 64 primer combinations supplied in the kit were previously tested on a single $C$. intestinalis specimen and AFLP fingerprints visualized with ethidium bromide staining. The efficacy of primer combinations was evaluated based on the sharpness, number, and size of fingerprint bands. Fifteen primer combinations that generated discrete bands ranging from 100 to $500 \mathrm{bp}$ were chosen for the present study (Supplemental Table 1). The preamplification step was performed for 20 cycles as follows: $94^{\circ} \mathrm{C}$ for $30 \mathrm{sec}, 56^{\circ} \mathrm{C}$ for $60 \mathrm{sec}$, and $72^{\circ} \mathrm{C}$ for $60 \mathrm{sec}$. Prior to PCR, the EcoRI primer was end-labeled with [gamma- ${ }^{32} \mathrm{P}$ ] ATP $(3,000 \mathrm{Ci} / \mathrm{mmol}$; Amersham Bioscience), and the selective amplification was performed with a touchdown program for 13 cycles: $94^{\circ} \mathrm{C}$ for $30 \mathrm{sec}, 65^{\circ} \mathrm{C}$ for $30 \mathrm{sec}$, and $72^{\circ} \mathrm{C}$ for $60 \mathrm{sec}$, with a $0.7^{\circ} \mathrm{C}$ decrease of annealing temperature each cycle, followed by 23 cycles of amplification at $94^{\circ} \mathrm{C}$ for $30 \mathrm{sec}, 56^{\circ} \mathrm{C}$ for $30 \mathrm{sec}$, and $72^{\circ} \mathrm{C}$ for $60 \mathrm{sec}$. A total of $4 \mu \mathrm{l}$ of each sample was loaded on a $6 \%$ denaturing polyacrylamide gel and run for $2 \mathrm{hr}$ at $40 \mathrm{~W}$. AFLP fingerprints were visualized by autoradiography using X-ray film (Kodak Photo Film Co. LTD). PCR was carried out two or three times using the same pre-amplified reactions, and only reproducible markers were used for further analyses. To clearly separate some close markers, electrophoresis was performed for $1.5 \mathrm{hr}$ at $55 \mathrm{~W}$. Markers were manually scored for presence or absence of bands with comparable gel mobility. Each marker was named B (British) or N (Neapolitan), followed by the number of the primer combination and by the product number in decreasing order of fingerprint size. An initial "d" was added to the names of "distorted" markers (see below).

\section{Linkage analyses}

Genotyping was performed on each separate data set for the maternal British and the paternal Neapolitan markers. To allow the detection of linkage in repulsion phase, duplicate data sets were prepared (Grattapaglia and Sederoff, 1994). Markers were classified into Mendelian or non-Mendelian (= "distorted") markers using the chi-square test for backcross manner $(1: 1$ ratios) at $P<0.05$. Extremely distorted markers $(P<0.01)$ were discarded. Only Mendelian markers were used for initial framework maps developed with MAPL98 (Ukai et al., 1991) and Mapmaker II software (Lander et al., 1987). Grouping was carried out manually using the nearest neighboring loci method in MAPL98, with a LOD score of 3.00 and a recombination frequency threshold of 0.26 . Unlinked markers below the threshold values were mapped using the TwoPoint/Near and MultiPoint/TRY commands in Mapmaker II with lower thresholds ( $L O D=1.44$ and recombination frequency $=0.32$ ).

To construct the final maps, non-Mendelian markers were mapped onto the initial framework maps in the same way as for Mendelian markers. Some non-Mendelian markers that disintegrated the initial framework were discarded from further analyses. Genetic distances were estimated using the Kosambi function. The maps derived from the two parents were not merged into an integrated map, by using markers showing intercross manner, because of the limited number of F1 progeny analyzed.

Table 1. Summary of the number of AFLP markers generated from each primer set

\begin{tabular}{|c|c|c|c|c|c|c|c|c|c|c|}
\hline & \multicolumn{3}{|c|}{ Common Markers } & \multicolumn{3}{|c|}{ British Markers } & \multicolumn{3}{|c|}{ Neapolitan Markers } & \multirow[b]{2}{*}{ total } \\
\hline & Mono & Mendelian & Distorted & Mono & Mendelian & Distorted & Mono & Mendelian & Distorted & \\
\hline AFLP\#01 & 1 & 0 & 1 & 2 & 6 & 14 & 3 & 5 & 3 & 35 \\
\hline AFLP\#02 & 2 & 0 & 3 & 1 & 21 & 19 & 3 & 4 & 8 & 61 \\
\hline AFLP\#03 & 1 & 2 & 3 & 1 & 21 & 7 & 6 & 6 & 0 & 47 \\
\hline AFLP\#04 & 3 & 3 & 10 & 1 & 18 & 18 & 3 & 18 & 10 & 84 \\
\hline AFLP\#05 & 1 & 1 & 2 & 2 & 13 & 11 & 8 & 5 & 6 & 49 \\
\hline AFLP\#06 & 1 & 1 & 1 & 1 & 8 & 4 & 2 & 6 & 2 & 26 \\
\hline AFLP\#07 & 2 & 3 & 7 & 1 & 21 & 15 & 3 & 7 & 6 & 65 \\
\hline AFLP\#08 & 1 & 3 & 4 & 0 & 12 & 9 & 1 & 3 & 9 & 42 \\
\hline AFLP\#09 & 1 & 1 & 0 & 3 & 16 & 14 & 3 & 4 & 2 & 44 \\
\hline AFLP\#10 & 0 & 0 & 0 & 1 & 11 & 2 & 2 & 4 & 1 & 21 \\
\hline AFLP\#11 & 1 & 0 & 1 & 0 & 8 & 6 & 4 & 3 & 2 & 25 \\
\hline AFLP\#12 & 5 & 0 & 1 & 7 & 8 & 9 & 5 & 5 & 2 & 42 \\
\hline AFLP\#13 & 3 & 1 & 1 & 4 & 16 & 15 & 6 & 4 & 11 & 61 \\
\hline AFLP\#14 & 2 & 1 & 1 & 2 & 15 & 7 & 6 & 2 & 7 & 43 \\
\hline AFLP\#15 & 4 & 3 & 1 & 1 & 7 & 2 & 2 & 2 & 3 & 25 \\
\hline total & 28 & 19 & 36 & 27 & 201 & 152 & 57 & 78 & 72 & 670 \\
\hline
\end{tabular}

Primer combinations for each primer set are indicated in Supplemental Table1. Mono, Mendelian, and Distorted mean Monomorphic, Mendelian Polymorphic, and Distorted Polymorphic markers, respectively. 


\section{Estimation of genome length and coverage}

Measures of the total lengths for the initial framework and final maps (Goi and Gof) were based on the sum of each linkage group. Estimation of genome length $(\mathrm{Ge})$ and expected map coverages (Cei and Cef) were estimated according to Tani et al. (2003). A minimum LOD score of 3.08 was chosen to estimate the genome length.

\section{Conversion of AFLP to STS}

The target band was cut out from the dried polyacrylamide ge and dissolved in $200 \mu \mathrm{l}$ of TE buffer for one hour, followed by incubation at $80^{\circ} \mathrm{C}$ for $15 \mathrm{~min}$. The mix was centrifuged at $15 \mathrm{~K} \mathrm{rpm}$ for
5 min, and supernatant was placed into another tube. The PCR product was precipitated with $1 / 50$ volume of $5 \mathrm{M} \mathrm{NaCl}$ and glycogen in ethanol, and dissolved in $15 \mu \mathrm{l}$ of sterile water. Of this, $5 \mu \mathrm{l}$ were used for re-amplification with the same touchdown program for AFLP in a total volume of $50 \mu \mathrm{l}$. The PCR product was collected using a low melting point gel and cloned using TOPO TA (Invitrogen). Insert fragments of 14 clones were checked, and four of them were sequenced. If all four clones did not have homologous sequences, the entire process was repeated. Markers that represented multiple sequences, even after double experiments, were discarded from the map and further analyses.

Sequences were analyzed and determined for scaffold location

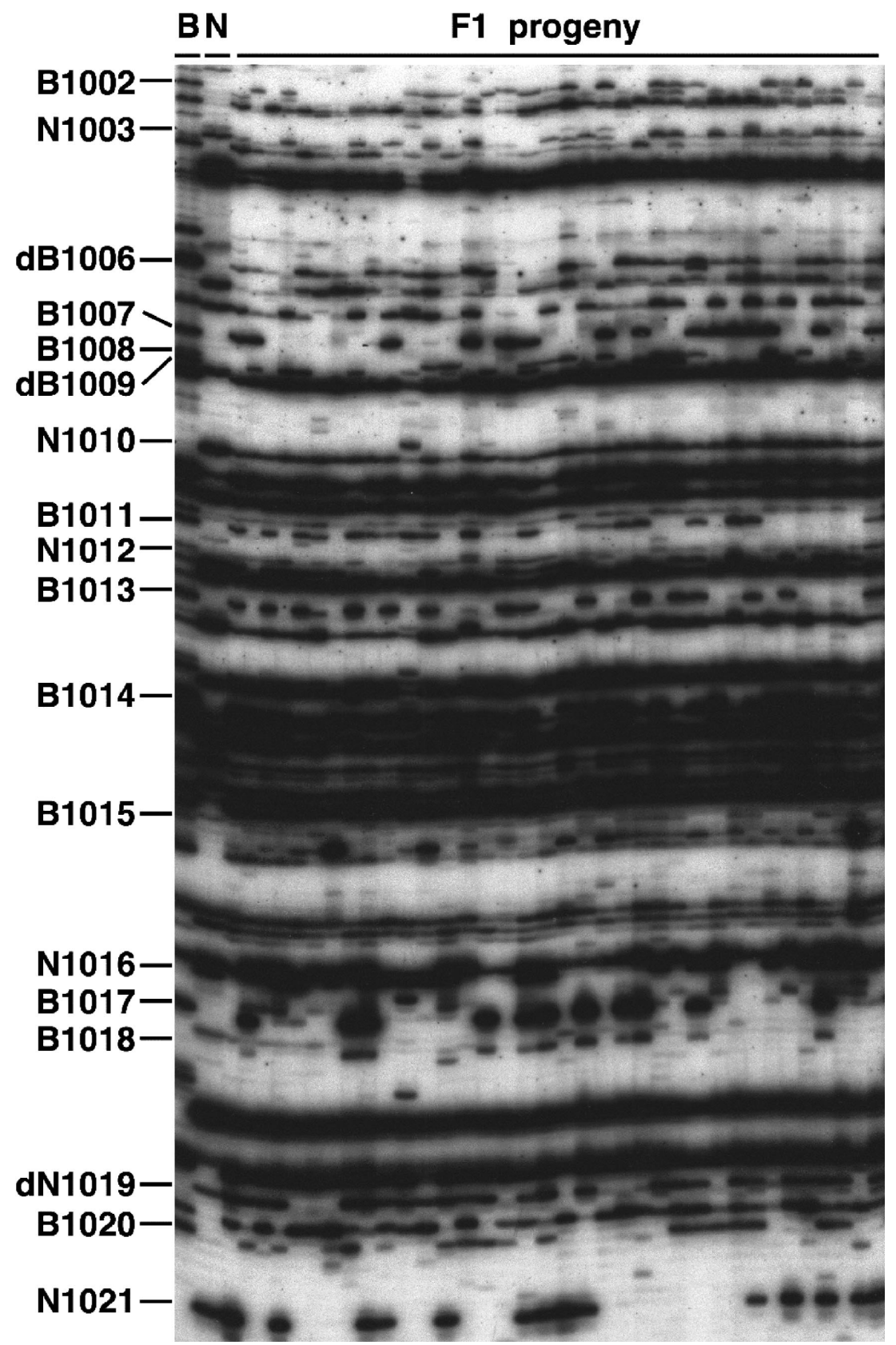

Fig. 1. An example of AFLP gel electrophoresis using primer combination No. 10. The first two lanes represent the two parents (B, British; N, Neapolitan), and the other lanes are fingerprints of $\mathrm{F} 1$ progeny. Reproducible markers from several experiments were analyzed, and only polymorphic markers are indicated in this figure, except for one example of a monomorphic marker, N1010. A marker located above the gel image, B1001, is not shown due to size limitation of the figure. One marker, B1018, was analyzed because repeatability was confirmed although the parental band is too weak to be seen in this figure. 
using a genome browser, $C$. intestinalis $v 1.0$ by the Joint Genome Institute (JGl; http://genome.jgi-psf.org/ciona4/; Dehal et al., 2002). A sequence found in a unique scaffold was presumed to correspond to a single site. Positions on chromosomes for the corre- sponding scaffolds were confirmed using the Ghost database (http:/ /hoya.zool.kyoto-u.ac.jp/chromosomeall.html; Satou et al., 2005). The STS sequences were deposited into GenBank as B607 (AY879054), B608 (AY879055), and B0606d2 (DQ178661).

Table 2. Estimations of genome lengths and map coverages

\begin{tabular}{lll}
\hline & the British map & the Neapolitan map \\
\hline $\begin{array}{l}\text { Genome length } \\
\text { Observed } \\
\text { Gof (Goi) }[\mathrm{cM}]\end{array}$ & & \\
Estimated & $4218.9(2624.5)$ & $2086.9(980.8)$ \\
$\quad$ Ge cM & 6199.8 & 3206.0 \\
$\quad$ Lower bound [cM] & 5929.4 & 2908.2 \\
Higher bound [cM] & 6448.5 & 3457.2 \\
Genome coverage for Cef $(\mathrm{Cel})[\%]$ & $74.1(51.7)$ & $69.2(45.6)$ \\
\hline
\end{tabular}

Abbreviations: Gof, total observed genome length of the final map; Goi, total observed genome length of the initial framework map; Ge, Estimated genome length; Cef, Coverage of estimated genome length of the final map; Cei, Coverage of estimated genome lenght of the initial map.
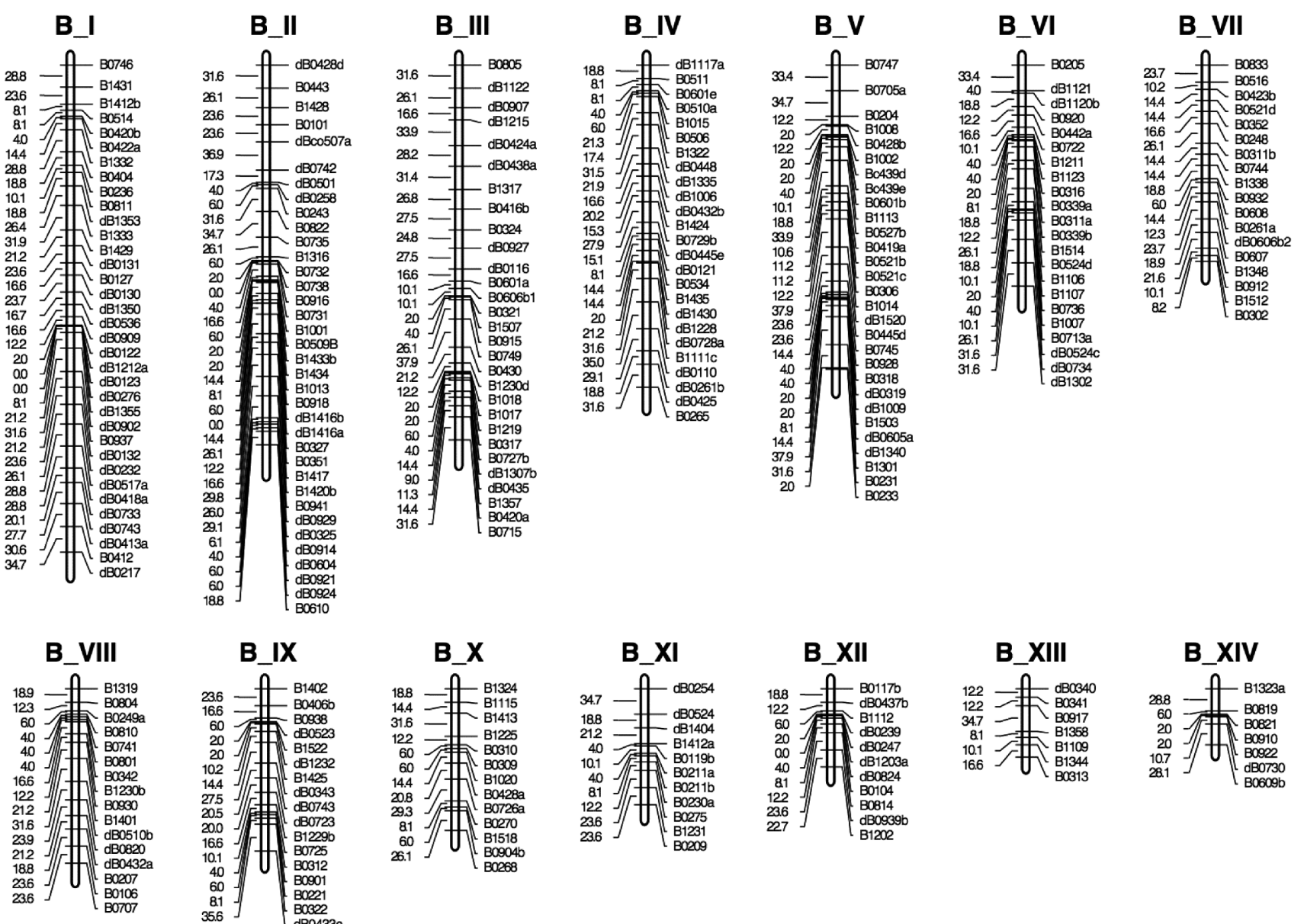

\section{B_IX}

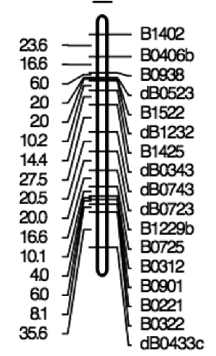

B $\mathbf{X}$

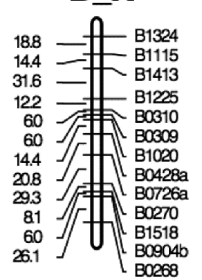

B_XI

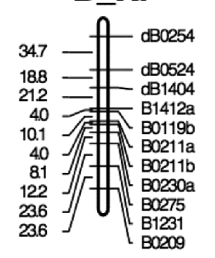

B XII

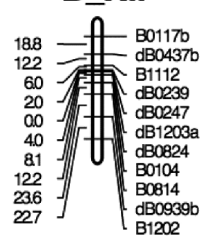

B XIII

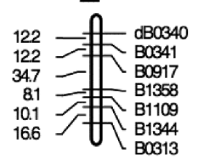

B XIV

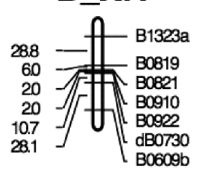

Fig. 2. Genetic linkage map of the ascidian Ciona intestinalis derived from the British individual. We mapped 276 AFLPs covering $4218.9 \mathrm{cM}$ (Kosambi mapping function), using 50 F1 progeny. The 14 linkage groups were ordered and numbered on the basis of size. Map distance of each interval is described in centimorgans. Each marker was named B (British), followed by the number of the primer combination and by the product number in decreasing order of fingerprint size. "Distorted" markers are indicated with the initial letter "d". 


\section{RESULTS}

\section{Primer combinations and AFLP markers}

To begin with, primer screening was performed without radioisotope labeling, and each combination was evaluated visually as described in Materials and Methods (Supplemental Table 1). Fifteen AFLP primer combinations generated 670 markers in total, ranging in number from 21 to 84 with
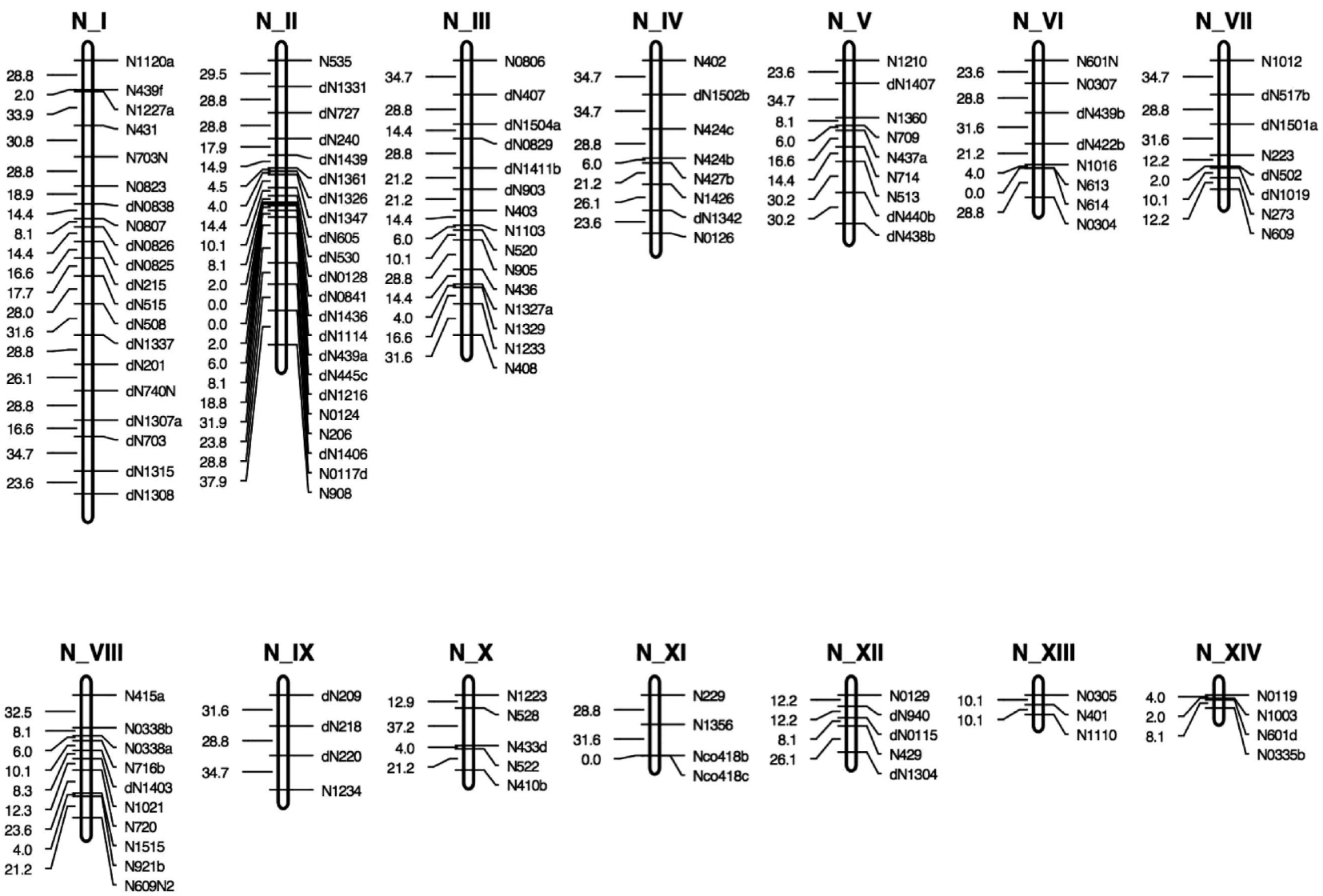

Fig. 3. Genetic linkage map of $C$. intestinalis derived from the Neapolitan individual. A total of 125 AFLPs were mapped, using the same cross panel shown in Fig. 1, covering 2086.9 cM. Each N marker (Neapolitan) was named as in the British map (Fig. 2).

Table 3. Distribution of markers and lengths for each linkage group in the final maps

\begin{tabular}{|c|c|c|c|c|c|}
\hline \multicolumn{3}{|c|}{ the British map } & \multicolumn{3}{|c|}{ the Neapolitan map } \\
\hline LG & Length (cM) & Total no. loci & $L G$ & Length (cM) & Total no. loci \\
\hline B_I & 657.0 & 35 & N_I & 432.7 & 20 \\
\hline B_II & 524.0 & 36 & N_II & 320.4 & 22 \\
\hline B_III & 509.0 & 29 & N_III & 275.0 & 15 \\
\hline B_IV & 438.5 & 25 & N_IV & 175.1 & 8 \\
\hline B_V & 416.2 & 29 & N_V & 163.8 & 9 \\
\hline B_VI & 305.1 & 22 & N_VI & 138.1 & 8 \\
\hline B_VII & 268.3 & 18 & N_VII & 131.7 & 8 \\
\hline B_VIII & 242.0 & 16 & N_VIII & 126.1 & 10 \\
\hline B_IX & 223.2 & 17 & N_IX & 95.1 & 4 \\
\hline B_X & 193.9 & 13 & N_X & 75.3 & 5 \\
\hline B_XI & 160.4 & 11 & N_XI & 60.5 & 4 \\
\hline B_XII & 109.8 & 11 & N_XII & 58.7 & 5 \\
\hline B_XIII & 93.9 & 7 & N_XIII & 20.3 & 3 \\
\hline B_XIV & 77.6 & 7 & N_XIV & 14.1 & 4 \\
\hline total & 4218.9 & 276 & total & 2086.9 & 125 \\
\hline
\end{tabular}


an average of 44.2 per primer combination (Table 1). Of these, 380 (56.7\%) and 207 (30.9\%) markers were derived from the British and Neapolitan specimens, respectively. The remaining 83 (12.4\%) AFLP markers were in common, indicating that the genetic difference between the two populations was effective and useful for genetic analyses (Fig.1). The number of British markers was almost twice that of Neapolitan ones, and this tendency was observed for every primer combination, with statistical significance $(P<0.01$, chi-square test). Of the parent-specific markers, 303 British and 134 Neapolitan polymorphic markers were considered for linkage analyses after extremely distorted markers were discarded.

\section{Genetic maps}

Grouping using the nearest neighboring loci method in MAPL98 provided reasonable results, whereas grouping based only on the LOD threshold and the frequency values resulted in fragmentation of linkage groups. Ciona intestinalis is hermaphroditic, with 14 chromosomes per haploid genome (Shoguchi et al., 2005); thus, we expected the markers to be distributed over 14 linkage groups. Using only Mendelian markers in the initial framework maps, British markers grouped into 16 linkage groups, and Neapolitan markers into 8 linkage groups, 2 triplets, and 2 doublets, covering 2624.5 and $980.8 \mathrm{cM}$, respectively (Goi in Table 2). Twelve (6.0\%) of 201 British and 14 (17.9\%) of 78 Neapolitan Mendelian markers remained unlinked. In total, $85.0 \%$ (British) and 67.9\% (Neapolitan) of marker linkages showed tight linkage (LOD>3.0). The average interval in genetic distance was $15.1 \mathrm{cM}$ in the British and $18.5 \mathrm{cM}$ in the Neapolitan initial framework maps. From the partial linkage data of the initial framework maps, we calculated genome length $(\mathrm{Ge})$ in genetic distance as $6199.8 \mathrm{cM}$ and $3206.0 \mathrm{cM}$, respectively, for the British and Neapolitan maps. Comparably, coverage of genome length (Cei) was $51.7 \%$ (British) and $45.6 \%$ (Neapolitan).

In the final maps including non-Mendelian markers (distorted markers; see Materials and Methods), 276 British markers grouped into 14 linkage groups covering $4218.9 \mathrm{cM}$ (Gof in Table 2 and Fig. 2). Likewise, 125 Neapolitan markers generated 13 linkage groups and one triplet, corresponding to $2086.9 \mathrm{cM}$ (Gof in Table 2 and Fig. 3). While some Mendelian markers were unlinked in the initial maps, they could be assigned to the final maps by increasing the total number of markers with non-Mendelian loci. Only 27 $(8.9 \%)$ of 303 markers were unlinked in the final British map, and nine $(6.7 \%)$ of 134 in the Neapolitan one. Lengths of linkage groups in the final maps varied to a large extent, from 77.6 to $657.0 \mathrm{cM}$ (British), and from 14.1 to $432.7 \mathrm{cM}$ (Neapolitan) (Table 3). Coverage (Cef) was estimated as 74.1 and $68.7 \%$ for the British and Neapolitan total maps, respectively (Table 2). Since any small chromosomes should be at least $50 \mathrm{cM}$ in size due to an obligate chiasma (Henderson, 1963), two linkage groups in the Neapolitan map, LG_N_XIII (37.8 cM) and LG_N_XIV (19.3 cM), can be further expanded. Based on the coverages, approximately 370 British and 200 Neapolitan markers are required to cover the whole genome in the two final maps.

Some loci displayed cluster distributions, with higher marker densities than those for the entire final maps (Fig. 2 and 3). For instance, the average interval size was $5.2 \mathrm{cM}$ in the lower part of LG_B_III and $5.4 \mathrm{cM}$ in a central region of LG_N_II. Some clusters may correspond to centromeres, known to be recombinationally suppressed (Stephan and Mitchell, 1992), such as the region around the marker B0211a in LG_B_XI, which was composed of Mendelian markers at high density. Conversely, clusters of distorted markers were observed in some linkage groups, such as in the lower part of LG_B_II and LG_B_III. Interestingly, distorted markers occurred in the central and slightly upper region of LG_N_II, around markers dN530 and dN1326, and in LG_B_II, around markers B1011 and B0732, suggesting that the two linkage groups could be homologous. Such observations might be associated with biological traits rather than experimental artifacts (see Discussion). However, this hypothesis does not always apply, since distorted markers occurred over broad regions (e.g., LG_B_I, LG_B_IV, and LG_N_I).

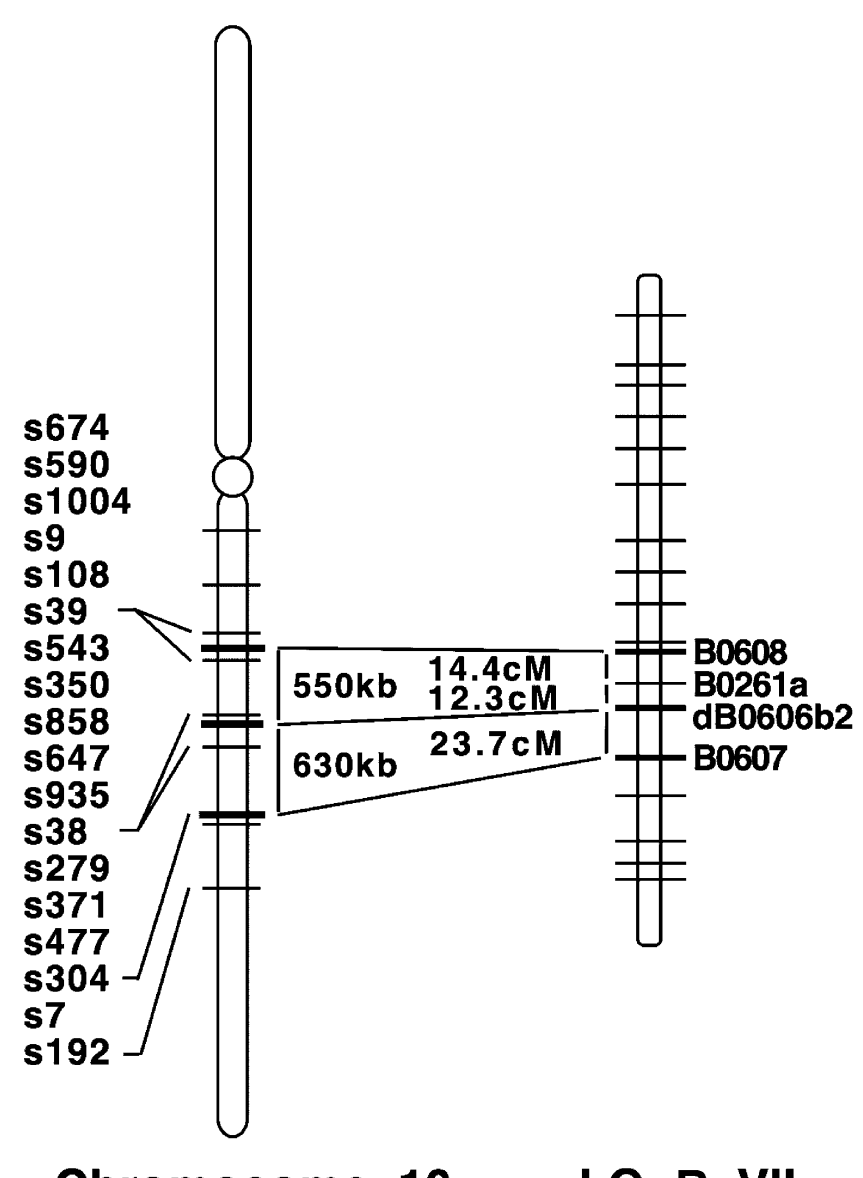

\section{Chromosome_10 LG_B_VII}

Fig. 4. Correlation between a chromosome No. 10 (referring to the Ghost database) and linkage group B_VII (Fig. 1). Three STS markers, B608, dB606b2, and B607 (Supplemental Table 2), corresponded to three scaffolds (bold lines in chromosome 10) in the same order (bold lines in LG_VII). A marker lying among the three, B0261a, was too short a fragment to convert. Length of the linkage group was adjusted to approximately $60 \%$ of the chromosome size, since coverage of the linkage group was estimated at that ratio with the present markers. 


\section{Correlation of physical to genetic distance}

Considering that the physical genome size is approximately $156 \mathrm{Mb}$ (Dehal et al., 2002), the average ratios of physical to genetic distance were calculated to be $25.2 \mathrm{~kb} /$ $\mathrm{cM}$ for the British map and $48.7 \mathrm{~kb} / \mathrm{cM}$ for the Neapolitan map. To directly calculate this correlation, the physical map was compared with the linkage maps through STS markers converted from AFLP markers. Three AFLPs on LG_B_VII, which showed a similar average interval and no clustering in the entire map, were converted to determine corresponding physical scaffolds (Supplemental Table 2). The three corresponding STSs were found to be located on chromosome 10 (sensu the Ghost database), indicating that linkage group B_VII corresponds to chromosome 10. The genetic distance corresponded to $50.4 \mathrm{cM}$, while the physical distance was $1.18 \mathrm{Mb}$ (Fig. 4), leading to a correlation of genetic to physical distance of $23.1 \sim 45.2 \mathrm{~kb} / \mathrm{cM}$. Based on this range of values and the estimated genome length $\mathrm{Ge}$, the entire genome length was calculated as 137.1 268.3 $\mathrm{Mb}$, which is indeed in agreement with $C$. intestinalis genome length (156 Mb). Hence, the observed recombination values are realistic.

\section{DISCUSSION}

\section{Genetic backgrounds and chromosome structures}

The AFLP markers obtained were sufficient in number to construct genetic maps, indicating that genetic differences between the two geographically separate populations were sufficient to provide new markers, and that heterozygosities of the parents were high enough to use the F1 pseudo-testcross strategy. We note that the estimated genome size $(\mathrm{Ge})$ of the British map was larger. One possible explanation is that the recombinant ratio varies between female and male gametes. In insects and vertebrates, female gametes show higher recombination than male ones (Yasukochi, 1998; Singer et al., 2002), and a similar phenomenon might occur in $C$. intestinalis as well.

Map analysis revealed some clustered regions consisting of distorted markers, which may be linked with specific biological functions. The genetic backgrounds of the two populations are so distant (as supported by the AFLP data) that genetic incompatibilities could have arisen at various developmental stages. Further, embryos can be negatively selected by environmental factors, for instance, salinity and temperature (Dybern, 1965, 1967). Indeed, British offspring grew poorly in our facility, whereas F1 crosses between British and Neapolitan gametes grew successfully and produced F2 progenies. These observations suggest that the genetic background influences embryonic development and growth, which might be responsible for the observed distortions (Harushima et al., 2001; Myburg et al., 2004). On the other hand, tolerance limits to environmental parameters are so variable that it is hard to define them exactly.

The diploid karyotype of this species contains 20 metacentric and eight submetacentric or subtelocentric chromosomes (Shoguchi et al., 2005). In the present study, we observed several correlations between the karyotype and the linkage groups. Considering that the density of genetic markers likely reveals the presence of a centromere, clusters on LG_B_III, IV, VIII, and IX could reflect submetacentric chromosomes, and LG_B_XI and XIV could be meta- centric. The very large chromosome No. 1 (sensu Shoguchi et al., 2005) might occur in both maps as the largest linkage groups, LG_B_I and LG_N_I, if genetic distance were to reflect physical distance.

\section{Recombination ratio in Ciona intestinalis}

Linkage analysis of AFLP markers showed that high recombination occurred not in specific genomic sub-regions, but in the entire genome of $C$. intestinalis, indicating that a high recombinant frequency is a normal feature of this genome. The ratio of physical to genetic distance $(90 \mathrm{~kb} / \mathrm{cM})$ was also low in the Fu/HC locus of the tunicate Botryllus schlosseri (De Tomaso and Weissman, 2003), suggesting that high recombination may be a common trait of ascidians. It is known that the ratio of physical length to genetic distance has increased during evolution (Catcheside, 1977). For example, this value is 2.32 and $3.84 \mathrm{~kb} / \mathrm{cM}$ in $E$. coli (Blattner et al., 1997) and yeast (Bussey et al., 1997), and 210, 280, 575, and $770 \mathrm{~kb} / \mathrm{cM}$ in Arabidopsis (Peters et al., 2001), Drosophila (Merriam et al., 1991), rice (Harushima et al., 2001) and human (Yu et al., 2001), respectively. Herein, the ratio was estimated to be about $25 \sim 49 \mathrm{~kb} / \mathrm{cM}$ in $C$. intestinalis.

High recombination might be associated with chromosome size and rearrangements. In fact, $C$. intestinalis, with a genome size comparable to that of Arabidopsis and Drosophila, has 14 pairs of chromosomes, whereas the latter two species have only five and three pairs of chromosomes, respectively (Arabidopsis Genome Initiative, 2000; Adams et al., 2000). Smaller chromosomes should result in a higher average frequency of recombination simply due to chiasma frequency, as in plants (Rees and Durrant, 1986). Thus, the recombinant ratio in Ciona should be higher than that in Arabidopsis and Drosophila, irrespective of overestimation due to dominant markers. Further, evidence of variation in the size of three chromosomes containing ribosomal DNA (rDNA) repeats (Shoguchi et al., 2005) suggests that such repetitive elements are associated with high recombination and genomic mobility.

Together with the effects of large effective population size (Dehal et al., 2002), the recombination rate could also justify the genetic polymorphisms of this species, since crossing-over is known to generate mutations (Lercher and Hurst, 2002; Filatov and Gerrard, 2003). This hypothesis could also explain the persistent polymorphisms occurring in inbreeding lines (Kano et al., 2001).

\section{Applications of the maps in Ciona genetics}

The main advantage of the $\mathrm{F} 1$ pseudo-testcross strategy using AFLP markers was quick construction of genetic maps. The applicative value of co-dominant markers is counterbalanced by efforts required to develop them in cases of a high degree of polymorphism and the absence of laboratory strains, such as in C. intestinalis. However, AFLP mapping combined with STS conversion was effective in primary genetic mapping, and then in generating co-dominant markers. Originally derived from the multiallelic polymorphisms due to insertion/deletion and from SNPs at the population and individual levels, STSs can be converted to universal co-dominant markers. In this sense, most marine invertebrates that exhibit a high degree of polymorphism 
and large broods, such as the amphioxus Branchiostoma floridae and the sea urchin Strongylocentrotus purpuratus, carry suitable traits for construction of genetic maps using F1 pseudo-crossing, despite the hurdles of culturing and breeding. Also for these species, whose genome projects are progressing, linkage maps will be crucial for assembling genome fragments, in combination with other physical mapping strategies such as clone-array pooled shotgun sequencing (CAPSS), FISH (Shoguchi et al., 2004), and integrated databases of genomic and cDNA data (e.g., Ghost database; Satou et al., 2005).

In the present study, our genetic maps revealed the genome structure of the ascidian $C$. intestinalis and provided information on genetic loci using widely distributed AFLP markers. The high recombinant ratio may be a trait advantageous for enabling us to carry out fine mapping with a relatively small number of progeny. Information derived from STS markers will contribute to the integration of genetic and physical maps. The cross-panel material gives increasingly numerous AFLP markers, providing a STS resource to study mutant loci. Investigation of distorted genomic regions, which could be associated with physiological events and lethal loci, together with analysis of quantitative trait loci (QTL) associated with growth rate, will help in the establishment of stable laboratory strains.

\section{ACKNOWLEDGMENTS}

We express our special thanks to Dr. R. De Santis and all members of the laboratory of Cell Biology, where most of this work was undertaken; Dr. M. Branno for giving permission to use a thermo-stable room in her laboratory; P. Cirino, A. Monte, A. Macina, C. Zazo, the Marine Resource for Research Service, and the Fishery Service of SZN for care and supplies of ascidians; E. Biffali and the Molecular Biology Service of SZN for reagents and sequencing; F. Esposito for providing living diatoms and algae to feed Ciona; Dr. S. M. Shimeld (University of Oxford, UK) for shipping us British animals many times; E. Shoguchi for sharing results about the karyotype before publication; and Dr. K. Naruse (University of Tokyo, Japan), Dr. M. Hill (Stanford University, USA), and several other colleagues for discussions and comments on the manuscript. S.K. was a Research Fellow of the Japan Society for the Promotion of Science (JSPS), with research grant No. 60048. This research was supported by a Grant-in-Aid from the Ministry of Education, Culture, Sports, Science and Technology of Japan (MEXT) to N.S. (12202001), a Key Action grant from the European Community (EC) to P.S. and S.K. (QLK3-CT2001-1890) and a Cluster grant from the Ministero dell'Istruzione, dell'Università $e$ della Ricerca (MIUR) to P.S. (C08c-24).

\section{REFERENCES}

Adams MD, Celniker SE, Holt RA et al. (2000) The genome sequence of Drosophila melanogaster. Science 287: 2185-2195

Arabidopsis Genome Initiative (2000) Analysis of the genome sequence of the flowering plant Arabidopsis thaliana. Nature 408: $796-815$

Awazu S, Sasaki A, Matsuoka T, Satoh N, Sasakura Y (2004) An enhancer trap in the ascidian Ciona intestinalis identifies enhancers of its Musashi orthologous gene. Dev Biol 275: 459-472

Blattner FR, Plunkett G 3rd, Bloch CA et al. (1997) The complete genome sequence of Escherichia coli K-12. Science 277: 1453-1474

Boffelli D, Weer CV, Weng L, Lewis KD, Shoukry MI, Pachter L, Keys DN, Rubin EM (2004) Intraspecies sequence comparisons for annotating genomes. Genome Res 14: 2406-2411
Bussey H, Storms RK, Ahmed A, Albermann K, Allen E, Ansorge W, Araujo R, Aparicio A, Barrell B, Badcock K (1997) Saccharomyces cerevisiae chromosome XVI. Nature 387: 103-105

Catcheside DG (1977) The Genetics of Recombination, Ed by A Edward, University Park Press, London, pp 172

Cirino P, Toscano A, Caramiello D, Macina A, Miraglia V, Monte A (2002) Laboratory culture of the ascidian Ciona intestinalis (L.): a model system for molecular developmental biology research. Mar Mod Elec Rec. Available at http://www.mbl.edu/Biological Bulletin/MMER/cirino/CirTit.html

De Tomaso AW, Weissman IL (2003) Initial characterization of a protochordate histocompatibility locus. Immunogenetics 557: 480-490

Dehal P, Satou Y, Campbell RK et al. (2002) The draft genome of Ciona intestinalis: insights into chordate and vertebrate origins. Science 298: 2157-2167

Deschet K, Nakatani Y, Smith WC (2003) Generation of CiBrachyury-GFP stable transgenic lines in the ascidian Ciona savignyi. Genesis 35: 248-259

Dybern BI (1965) The life cycle of Ciona intestinalis (L.) f. typica in relation to the environmental temperature. Oikos 16: 109-131

Dybern BI (1967) The distribution and salinity tolerance of Ciona intestinalis (L.) f. typica with special reference to the waters around southern Scandinavia. Orphelia 4: 207-226

Filatov DA, Gerrard DT (2003) High mutation rates in human and ape pseudoautosomal genes. Gene 317: 67-77

Grattapaglia D, Sederoff R (1994) Genetic linkage maps of Eucalyptus grandis and Eucalyptus urophylla using a pseudo-testcross: mapping strategy and RAPD markers. Genetics 137: 11211137

Harushima Y, Nakagahra M, Yano M, Sasaki T, Kurata N (2001) A genome-wide survey of reproductive barriers in an intraspecific hybrid. Genetics 159: 883-892

Henderson SA (1963) Temperature and chiasma formation in Schistocerca gregaria II. Cytological effects at $40^{\circ} \mathrm{C}$ and the mechanism of heat induced univalece. Chromosoma 13: 437463

Jiang D, Munro E, Smith WC (2005) Ascidian prickle regulates both mediolateral and anterior-posterior cell polarity of notochord cells. Curr Biol 15: 79-85

Kano S, Chiba S, Satoh N (2001) Genetic relatedness and variability in inbred and wild populations of the solitary ascidian Ciona intestinalis revealed by arbitrarily primed polymerase chain reaction. Mar Biotechnol 31: 58-67

Lander ES, Green P, Abrahamson J, Barlow A, Daly MJ, Lincoln SE, Newburg L (1987) MAPMAKER: an interactive computer package for constructing primary genetic linkage maps of experimental and natural populations. Genomics 1: 174-181

Lercher MJ, Hurst LD (2002) Human SNP variability and mutation rate are higher in regions of high recombination. Trends Genet 18: 337-340

Li L, Guo X (2004) AFLP-based genetic linkage maps of the pacific oyster Crassostrea gigas. Mar Biotechnol 61: 26-36

Liu Z, Karsi A, Li P, Cao D, Dunham R (2003) An AFLP-based genetic linkage map of channel catfish Ictalurus punctatus constructed by using an interspecific hybrid resource family. Genetics 165: 687-694

Meksem K, Ruben E, Hyten D, Triwitayakorn K, Lightfoot DA (2001) Conversion of AFLP bands into high-throughput DNA markers. Mol Genet Genom 265: 207-214

Merriam J, Ashburner M, Hartl DL, Kafatos FC (1991) Toward cloning and mapping the genome of Drosophila. Science 254: 221225

Myburg AA, Griffin AR, Sederoff RR, Whetten RW (2003) Comparative genetic linkage maps of Eucalyptus grandis, Eucalyptus globulus and their F1 hybrid based on a double pseudo-backcross mapping approach. Theor Appl Genet 1076: 1028-1042 
Myburg AA, Vogl C, Griffin AR, Sederoff RR, Whetten RW (2004) Genetics of postzygotic isolation in Eucalyptus: whole-genome analysis of barriers to introgression in a wide interspecific cross of Eucalyptus grandis and E. globulus. Genetics 166: 14051418

Naruse K, Fukamachi S, Mitani H et al. (2000) A detailed linkage map of medaka, Oryzias latipes: comparative genomics and genome evolution. Genetics 154: 1773-1784

Peters JL, Constandt H, Neyt P, Cnops G, Zethof J, Zabeau M, Gerats $T$ (2001) A physical amplified fragment-length polymorphism map of Arabidopsis. Plant Physiol 127: 1579-1589

Peters JL, Cnops G, Neyt P, Zethof J, Cornelis K, Van Lijsebettens M, Gerats T (2004) An AFLP-based genome-wide mapping strategy. Theor Appl Genet 1082: 321-327

Procaccini G, Pischetola M, Di Lauro R (2000) PRIMER NOTE: Isolation and characterization of microsatellite loci in the ascidian Ciona intestinalis (L.). Mol Ecol 9: 1924-1926

Rees H, Durrant A (1986) Recombination and genome size. Theor Appl Genet 73: 72-76

Sasakura Y, Awazu S, Chiba S, Satoh N (2003) Germ-line transgenesis of the Tc1/mariner superfamily transposon Minos in Ciona intestinalis. Proc Natl Acad Sci USA 100: 7726-7730

Satoh N, Satou Y, Davidson B, Levine M (2003) Ciona intestinalis: an emerging model for whole-genome analyses. Trends Genet 19: $376-381$

Satou Y, Kawashima T, Shoguchi E, Nakayama A, Satoh N (2005) An integrated database of the ascidian, Ciona intestinalis: towards functional genomics. Zool Sci 22: 837-843

Shahjahan R, Hughes K, Leopold R, DeVault J (1995) Lower incubation temperature increases yield of insect genomic DNA isolated by the CTAB method. Biotechniques 19: 332-334

Shoguchi E, Ikuta T, Yoshizaki F, Satou Y, Satoh N, Asano K, Saiga $\mathrm{H}$, Nishikata T (2004) Fluorescent in situ hybridization to ascidian chromosomes. Zool Sci 21: 153-157
Shoguchi E, Kawashima T, Nishida-Umehara C, Matsuda Y, Satoh $\mathrm{N}$ (2005) Molecular cytogenetic characterization of Ciona intestinalis chromosomes. Zool Sci 22: 511-516

Singer A, Perlman H, Yan Y, Walker C, Corley-Smith G, Brandhorst B, Postlethwait J (2002) Sex-specific recombination rates in zebrafish Danio rerio. Genetics 160: 649-657

Sordino P, Belluzzi L, De Santis R, Smith WC (2001) Developmental genetics in primitive chordates. Philos Trans $R$ Soc Lond $B$ 356: 1573-1582

Stephan W, Mitchell SJ (1992) Reduced levels of DNA polymorphisms and fixed between-population differences in the centromeric region of Drosophila ananassae. Genetics 132: 10391045

Tani N, Takahashi T, Iwata H, Mukai Y, Ujino-Ihara T, Matsumoto A, Yoshimura K, Yoshimaru H, Murai M, Nagasaka K (2003) Consensus linkage map for sugi (Cryptomeria japonica) from two pedigrees, based on microsatellites and expressed sequence tags. Genetics 165: 1551-1568

Ukai Y, Ohsawa R, Saito A (1991) A package of microcomputer program for RFLP linkage mapping. Rice Genet Newslet 8: 155158

Vos $\mathrm{P}$, Hogers R, Bleeker M, Reijans M, van de Lee T, Hornes M, Frijters A, Pot J, Peleman J, Kuiper M (1995) AFLP: a new technique for DNA fingerprinting. Nucleic Acids Res 23: 44074414

Yasukochi Y (1998) A dense genetic map of the silkworm, Bombyx mori, covering all chromosomes based on 1,018 molecular markers. Genetics 150: 1513-1525

Yu A, Zhao C, Fan Y, Jang W, Mungall JA, Deloukas P, Olsen A, Doggett AN, Ghebranious N, Broman KW et al. (2001) Comparison of human genetics and sequence-based physical maps. Nature 409: 951-953

(Received September 19, 2005 / Accepted November 14, 2005) 